\section{$\underset{\substack{\text { hommes } \\ \text { \& migrations }}}{ }$}

\section{Hommes \& migrations}

Revue française de référence sur les dynamiques

migratoires

\section{9 | 2009}

L'Afrique en mouvement

\title{
Les pêcheurs du lac de Sélingué
}

Trajectoires et itinéraires de migration

\section{Salif Togola}

\section{OpenEdition}

1 Journals

Édition électronique

URL : http://journals.openedition.org/hommesmigrations/290

DOI : 10.4000/hommesmigrations.290

ISSN : 2262-3353

Éditeur

Musée national de l'histoire de l'immigration

Édition imprimée

Date de publication : 1 mai 2009

Pagination : 24-37

ISSN : 1142-852X

\section{Référence électronique}

Salif Togola, "Les pêcheurs du lac de Sélingué », Hommes \& migrations [En ligne], 1279 | 2009, mis en ligne le 29 mai 2013, consulté le 19 avril 2019. URL : http://journals.openedition.org/ hommesmigrations/290 ; DOI : 10.4000/hommesmigrations.290 


\section{Les pêcheurs du lac de Sélingué Trajectoires et itinéraires de migration}

Par Salif Togola, anthropologue, Faculté des Lettres, Langues, Arts et Sciences humaines, université de Bamako

La pêche au Mali est loin d'être une activité marginale, même si ce pays continental ne possède aucun débouché sur la mer. Avec la construction

du barrage hydroélectrique de Sélingué achevé en 1980, de nombreux pêcheurs nomades ont quitté leurs temitoires de pêche dans le Delta central du Niger pour s'installer autour du lac de retenue. Située à l'est de la frontière guinéenne, cette zone sert désormais de base à leur rayonnement, autant inteme qu'intemational. À la recherche des meilleures ressources en poissons, les pêcheurs de Sélingué migrent au fil de l'eau. 
La pêche artisanale s'est particulièrement dévéloppée dans les pays du tiers-monde. Malgré son caractère informel, elle représente une activité économique à part entière. Longtemps considérée comme une forme archaïque d'exploitation, depuis quelques décennies cependant, un ensemble de conditions se trouvent réunies pour qu'elle soit reconnue comme un véritable secteur d'activité et un objet de recherche à part entière. Au Mali, la flottille est essentiellement constituée de pirogues équipées d'engins d'un autre âge, mais bien adaptés aux conditions environnementales, au biotope et au comportement du poisson.

Entre 1970 et 1980, le Delta central du Niger ${ }^{(1)}$, principal foyer de production halieutique au Mali, connaît une grande sécheresse. Les communautés de pêcheurs quittent leurs territoires traditionnels de pêche, à la recherche de nouvelles opportunités. La migration des pêcheurs du delta répond aux aléas climatiques et va constituer désormais la base du système économique local. La fin de cette décennie coïncide avec l'ouverture du barrage de Sélingué dont la première pierre a été posée en 1976. Cette immense retenue d'eau avec ses multiples potentialités aura instantanément suscité la ruée des populations de tous les horizons du Mali pour exercer des activités d'ordre halieutique, agricole, etc. L'avènement du barrage va ainsi donner un souffle nouveau à la pêche. Des campements sont érigés le long des fleuves Sankarani et Wassoulou Balé, dont la confluence forme le lac de retenue. Ces campements constituent en quelque sorte l'armature des mouvements migratoires, tout en formant une série de points d'accueil en fonction desquels s'organisent les déplacements saisonniers des pêcheurs et les migrations proprement dites entre Sélingué et le Delta central ou d'autres lieux de pêche.

\section{La zone de Sélingué}

Le site de cette étude est Sélingué, une localité située dans le cercle de Yanfolila qui relève de la région de Sikasso, la troisième région économique du Mali. Logée dans le bassin du Sankarani, Sélingué est reliée, par une route bitumée de 140 kilomètres, à Bamako, la capitale de la République du Mali. Au niveau général, Sélingué faisait partie de l'ex-arrondissement de Kangaré qui se composait au départ de seize villages, dont Sélinkégny, un petit village malinké(2) situé tout juste derrière le barrage. Le nom "Sélingué" va désigner non seulement l'ex-arrondissement de Kangaré mais aussi toute la zone d'influence du barrage. Toutefois, consécutivement aux découpages territoriaux issus de la décentralisation en 1996, Kangaré dépend désormais de la commune rurale de Baya avec onze autres villages, 
des dizaines de hameaux de culture et des campements de pêche pour une population de 20391 habitants, selon le recensement démographique de 2004.

L'espace que désigne aujourd'hui "Sélingué" s'étend sur plusieurs villages qui, pour l'essentiel, relèvent du Baya, une aire socioculturelle malinké et dont il fut une province. En plus du Baya, le Mandé comptait plusieurs autres kafo ou territoires lignagers dont l'ensemble formait le mara, ou territoire politique de Soundjata. Chaque kafo, à son tour, était constitué de plusieurs familles (matricentrées) qui gardaient la chefferie du terroir de leurs ancêtres.

\section{Sélingué, point d'appui d'une pêche nomade}

En règle générale, les pêcheurs de Sélingué, d'origines et d'ethnies différentes, ont toujours constitué des communautés nomades. Leur sédentarisation n'annonce pas la fin de ce nomadisme. Ils sont arrivés dans cette zone en suivant le poisson et ils peuvent en repartir. Ces différents mouvements d'aller et de retour, dont les plus importants ont commencé depuis le Delta central, s'effectuent sur des trajectoires qui constituent leurs itinéraires de migration. Parler d'itinéraires, c'est parler du départ, du séjour et du retour, même s'il doit être entendu qu'il y a eu plusieurs départs, que le séjour peut aussi être un voyage et que le retour n'est jamais définitif. À la recherche du poisson, il arrive que le pêcheur abandonne son lieu de résidence d'origine pour partir avec ou sans sa famille, et ce en fonction des saisons, du niveau des prix de vente et de l'abondance de la ressource. La migration de pêche à Sélingué obéit à une logique d'ensemble qui est inséparable de cet ordre d'idées. Malgré une forte tendance à la sédentarisation ou à l'installation permanente dans des campements érigés à cet effet, les pêcheurs continuent d'orienter leurs déplacements au gré des cycles saisonniers, en direction de destinations choisies pour leur abondance de poissons. Ainsi, on peut considérer la pêcherie de Sélingué comme une phase ou un épisode dans leur processus de migration.

\section{Le parcours Delta Central - Sélingué - Delta central}

C'est la trajectoire de migration la plus fréquentée par les pêcheurs. Depuis le début des années quatre-vingt, les pêcheurs en provenance du Delta central ont emprunté cet itinéraire pour venir peupler les campements installés autour de la réserve d'eau du lac. Saisonniers au départ, les flux ont fini par se stabiliser. Les interactions entre le delta et la zone du barrage représentaient $52,2 \%$ des 
migrations en $1995^{(3)}$. Une décennie plus tard, la même tendance s'observe. Dans leur majorité (plus de 70\%), les chefs de famille qui ont répondu à nos questionnaires reconnaissent avoir rejoint Sélingué pour la première fois directement après avoir quitté leurs villages d'origine dans le Delta central du Niger, même si, par le passé, ils ont séjourné ailleurs dans le cadre de la pêche. Après la mise en eau du barrage, dans les années 1984-1985, ils ont eu des échos de la richesse en poisson de la zone. Ces informations ont provoqué une ruée populaire de centaines de pêcheurs vers Sélingué. "Nous avons appris que le barrage de Sélingué est ouvert et que le gouvernement permet à tous les pêcheurs de venir y pêcher." Ce discours ressort de toutes les conversations pour expliquer les raisons qui les ont poussés à quitter leur village d'origine.

\section{À la recherche des opportunités de pêche}

Un second itinéraire est constitué par des flux migratoires qui partent cette fois-ci de Sélingué vers le delta. Après un séjour de quatre à six mois chaque année, les premiers pêcheurs migrants regagnent leur village d'origine dans le Delta central. Les migrants saisonniers, qui représentent près de 30\% des pêcheurs de Sélingué, 
suivent cet itinéraire dans le cadre de la pêche. Ils font la navette entre Sélingué et le Delta central, à la recherche des meilleures zones de pêche. En réalité, ils ne sont fixés définitivement nulle part et leur trajectoire de migration peut s'étendre du delta jusqu'à Sélingué ou jusqu'au barrage de Manantali, situé à l'ouest du pays sur le fleuve Sénégal. Dans les campements, deux pêcheurs migrants saisonniers sur cinq ont séjourné au barrage de Manantali et sur d'autres pêcheries intérieures comme Bamako sur le fleuve Niger, Kolondièba, Kita ou Bougouni.

À Sélingué, il arrive que les pêcheurs effectuent des déplacements fréquents entre les différents campements érigés tout autour du lac. Ces déplacements sont, en général, le fait des saisonniers qui débarquent en grand nombre pendant la période de décrue (baisse des eaux du lac), avec ou sans leur famille, parmi d'autres pêcheurs déjà installés. Certains, ayant choisi de continuer leur trajectoire, peuvent étendre leur mouvement, à l'aide de la pirogue, jusqu'à l'amont du lac, vers la frontière guinéenne, en passant par les autres campements érigés le long du Sankarani et du Wassoulou Balé, et où ils peuvent séjourner. Sur les campements, leur séjour dure entre deux et six mois.

\section{La pêche, une affaire de saison}

Il est très difficile de recenser les migrants saisonniers pour réellement connaître leur itinéraire de migration car, du jour au lendemain, ils peuvent décider de se déplacer d'un lieu à un autre afin de suivre les mouvements du poisson. "Il y a deux semaines, jétais à Faraba et je compte, inch Allah, repartir à Tiéguékourouni le lundi prochain pour revenir ici vers la fin du mois", nous confiait Broulaye Sarré, un pêcheur saisonnier rencontré à Kangarékoro. Un autre, résidant au campement de Bélahdaga, résumait ses déplacements en ces termes : "Jai construit une case ici [Bélahdaga], mais cela ne m'empêche pas d'aller pêcher jusqu'à Yanfolila, Gualafara et Guélélenkoro chaque fois que l'eau du lac monte de trop par ici. Ainsi au bout de cinq à six mois, jai économisé un peu d'argent pour retourner chez moi."

Au début de l'hivernage, lorsque les premières pluies font monter les eaux du lac, ce qui correspond à la période de la basse production, une grande partie des pêcheurs migrants saisonniers (un sur cinq) commencent à se replier vers d'autres directions, notamment vers le Delta central ou des localités à l'intérieur du pays comme Kita, Kolondièba, Bougouni, où il y a moins de compétition dans l'accès à la ressource halieutique.

Depuis quelques années, face à la crise saisonnière due à la raréfaction de la ressource (entre novembre et février), même les pêcheurs installés de façon permanente dans 
un campement, adoptent de plus en plus la stratégie du nomadisme interne. Ils quittent alors les campements de résidence sans les femmes et les enfants pour aller à la pêche dans d'autres parties du lac. L'aval du lac, c'est-à-dire la partie comprise entre Faraba et la frontière guinéenne, est la destination prisée par les partants à cette période froide de l'année, car le niveau de l'eau y est plus bas. Les durées de séjour sont certes courtes (entre trois semaines et deux mois), mais ce genre de déplacement est pratiqué par deux pêcheurs sur cinq dans tous les campements visités.

\section{De Sélingué à l'extérieur du Mali}

Le processus de migration des pêcheurs, qui a débuté dans le delta, ne se termine pas toujours à Sélingué, qui a priori, semble être la destination finale. En réalité, un certain nombre de pêcheurs transitent par Sélingué, et poursuivent leur voyage jusque dans les pays limitrophes du Mali.

En raison de la proximité géographique de la Guinée avec la localité de Sélingué et surtout de Faraba, deuxième débarcadère de la zone après celui de Carrière, un bon nombre de pêcheurs, en suivant les bancs de poisson depuis Sélingué, se retrouvent en Guinée, y séjournent pendant trois à quatre mois avant de retourner à Sélingué. Ces déplacements hors des frontières nationales ne sont pas uniquement le seul fait des migrants saisonniers, puisque un chef de ménage sur dix environ indique avoir été en Guinée plus d'une fois pour un séjour dont la durée est comprise entre six et huit mois. Cet engouement des pêcheurs s'expliquerait par une certaine souplesse de la réglementation sur les pêcheries de ce pays. À titre d'exemple, la technique du barrage ${ }^{(4)}$ que la réglementation malienne interdit sur le lac de Sélingué serait permise en Guinée. Aussi cet itinéraire est-il fréquenté non seulement à cause de l'abondance de la ressource du pays hôte, mais également à cause des règles d'exploitation en vigueur sur le lac de Sélingué, que les pêcheurs trouvent de plus en plus rigides ${ }^{(5)}$.

\section{L'axe Côte d'Ivoire - Sélingué}

Aux campements de Carrière, Sélinkégny et Farabacoro, il y a des ménages de pêcheurs dont les chefs indiquent être venus à Sélingué directement depuis la Côte d'Ivoire. Leur installation est très récente, puisqu'elle remonte aux exactions exercées par les autorités ivoiriennes sur les communautés d'étrangers vivant en Côte d'Ivoire sous le régime d'Henri Konan Bédié. Les pêcheurs maliens immigrés 
en Côte d'Ivoire, plusieurs milliers en 1998, ont été spoliés de leurs biens et contraints de retourner dans leur pays d'origine. À cet égard, Sélingué a été un site d'accueil pour quelques-un de ces ménages expulsés, la majeure partie ayant été résorbée par le Delta central du Niger. Cet axe, un itinéraire de migration pour beaucoup, a constitué un chemin de retour au pays pour certains, même si parmi les pêcheurs rencontrés à Sélingué un bon nombre indique avoir été plus de deux fois en Côte d'Ivoire avant 1998 dans le cadre des migrations de pêche. Dans notre échantillon, deux pêcheurs sur trois, avant leur installation définitive à Sélingué, ont cumulé ces sorties hors du territoire d'origine de pêche. D'abord vers les pêcheries intérieures, puis au-delà des frontières du pays.

\section{Acquérir une expérience migratoire}

Le pêcheur migrant, tout au long de sa carrière, effectue des migrations dans plusieurs directions, et ce depuis qu'il est jeune, pour acquérir de l'expérience. Cette expérience migratoire est d'abord acquise par le jeune pêcheur sur des distances courtes ou moyennes, entre son campement d'origine et d'autres pêcheries de proximité. Ces campagnes saisonnières de pêche durent en moyenne entre quatre et six mois. 93\% de nos interlocuteurs déclarent avoir séjourné, pour la première fois, sur des pêcheries différentes de la leur, mais d'abord à l'intérieur du Delta central du Niger. Les jeunes pêcheurs de Ségou vont à Mopti, ou ceux de ces deux localités vont au nord (Tombouctou ou Gao). "Quand nous étions jeunes (21 et 19 ans) mon frère et moi partions, chaque année, de façon alternée, à Markala et Bamako. Je retournais au bout de quatre mois tandis que lui pouvait rester jusqu'à six mois voire sept. Cet itinéraire était notre trajectoire favorite jusqu'en 1987, où je suis venu m'installer ici à Faraba avec mes deux femmes."Les propos de ce pêcheur de 49 ans ne sont pas loin de confirmer ceux de deux jeunes pêcheurs migrants saisonniers.

Le premier est originaire du village de Samafléla, dans le cercle de Macina. À 25 ans à peine, célibataire, il a changé voici deux ans son ancien itinéraire de migration saisonnière pour venir à Sélingué, au campement de Tiéguékourouni. Auparavant, il suivait chaque saison le cours du fleuve Niger de Ségou, en passant par Mopti jusqu'à Tombouctou. Cette migration saisonnière se faisait à une période qui correspondait au temps de la reproduction dans les pêcheries du territoire d'où il est originaire. Ce long trajet était parcouru en six mois, au bout desquels il retournait dans son village pour l'exploitation des pêcheries lignagères. Actuellement, il a abandonné cet itinéraire au profit de celui de Sélingué, qui, selon lui, "est plus court et plus proche de Bamako où le poisson se vend à un prix un peu plus élevé". 
Le second pêcheur, rencontré au campement de Bozola, est célibataire, originaire de Koulikoro et âgé de 21 ans. Il a forgé son expérience migratoire sur les axes Koulikoro-Manantali et Koulikoro-Sélingué. Il n’a jamais pratiqué la pêche ailleurs au Mali, en dehors des barrages de Manantali et de Sélingué, contrairement à son père qui, selon lui, fréquente l'itinéraire Koulikoro-Bamako-Bougouni-Kita. Il a commencé à sortir de son village il y a trois ans. Pendant la première année (2002), il a passé seulement trois mois à Manantali. Depuis, il vient de son village chaque année à Sélingué, pendant cinq mois, pour faire la pêche. Il semble apprécier cet itinéraire en tant que saisonnier. "Si jarrive à économiser entre 100000 et 150000 CFA pendant ce laps de temps, je retourne

\section{Les migrations internationales de pêche correspondent au désir ardent et prestigieux de capturer le poisson hors des frontières nationales et d'amasser de l'argent.}

chez moi pour aider les miens aux travaux champêtres. L'année suivante, je reviens encore." La migration à Sélingué a été la première expérience migratoire pour seulement 4\% des pêcheurs interviewés, contre 3\% pour Manantali. Ce qui signifie que la majorité des pêcheurs migrants de Sélingué a séjourné d'abord, par le passé, ailleurs, sur d'autres campements, avant de venir s'installer à Sélingué.

\section{La migration au long cours}

Depuis bien longtemps, les pêcheurs maliens ont pris l'habitude de sortir pour aller très loin, jusqu'au lac Tchad, selon certaines sources. Les migrations internationales de pêche correspondent au désir ardent et prestigieux de capturer le poisson hors des frontières nationales et d'amasser de l'argent. Elles sont entreprises par des acteurs adultes qui ont déjà acquis beaucoup d'expérience dans la pratique de la pêche au pays d'origine. Ces longs parcours nécessitent l'abandon du pays par les pêcheurs pour une durée comprise entre un et sept ans. Les pêcheurs se rendent (cette fois-ci sans leur famille) dans presque tous les pays de l'Afrique occidentale, limitrophes du Mali : la Côte d'Ivoire, le Burkina Faso, la Guinée, le Ghana, la Mauritanie, le Niger, la Sierra Leone, le Liberia. En se référant aux expériences migratoires des pêcheurs rencontrés à Sélingué, un gros tiers d'entre eux a effectué deux ou trois sorties internationales depuis les années soixante avant de venir s'installer vers 1984-1985 à Sélingué à la faveur de la construction du barrage. Sur les 70 \% de chefs de ménage permanents, 40 \% se sont installés en 19841985, contre 30\% après 1986. Parmi les pays voisins les plus prisés par les 
pêcheurs migrants de Sélingué, c'est la Côte d'Ivoire avec son système lagunaire qui a abrité le plus gros des contingents avant les incidents de 1998. Sur les 36\% de pêcheurs ayant effectué des migrations internationales, 13\% déclarent s'y être rendus plus d'une fois. Après la Côte d'Ivoire, le Burkina Faso (notamment sur le barrage de Kompienga) a été le pays de destination de 10\% des pêcheurs ayant une expérience migratoire internationale, suivi du Ghana (le barrage de Kossu) avec 5\%, la Guinée 3\%, le Niger 2\%, la Mauritanie 2\% et la Sierra Leone 1\%.

Ainsi, un même pêcheur peut avoir effectué plusieurs migrations internationales successives dans différents pays. Pour les ménages de pêcheurs installés, Sélingué marque l'aboutissement des parcours qui ont constitué leurs expériences migratoires acquises lors de leur recherche des zones de pêche. C'est pourquoi, ils ne sont plus forcément candidats au départ vers d'autres zones de pêche. Ils se sont tout simplement sédentarisés.

\section{Des vies au fil de l'eau}

L'un de ces pêcheurs, âgé aujourd'hui de 67 ans et originaire de Kayo (Kologotomo) dans la région Ségou, Bakary est l'époux de trois femmes et père de treize enfants, soit sept filles et six garçons. Il est chef d'un ménage dont l'effectif s'élève au total à quaranteneuf individus, dont neuf actifs. Outre son propre foyer, que ses trois épouses partagent, il héberge sous son autorité ses deux frères

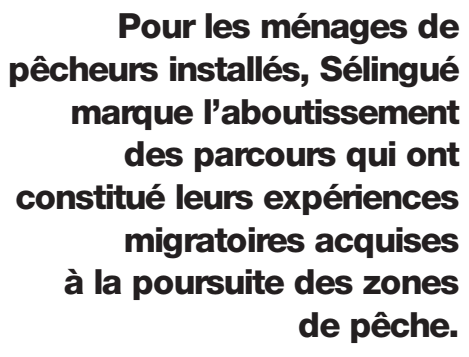
cadets, tous pêcheurs, et leurs épouses. Venu directement de son village pour la première fois à Sélingué dans le cadre des migrations de pêche en 1985, il reconnaît avoir acquis ses premières expériences migratoires à l'intérieur du Delta central à un âge très jeune. "Depuis mes 18 ans, je partais au nord (Gao, Tombouctou) chez les Issaboro (c'est le nom local donné au pêcheur en langue sonrai). Après quatre ou cinq mois, je retournais chez moi à Kayo auprès des parents, qui donnaient aussitôt la permission à mon frère aîné qui partait lui vers Mopti”. Il fréquente cet itinéraire pendant près de dix ans car, selon lui, "les jeunes pêcheurs sortent très rarement à l'extérieur du Mali." À la trentaine, il commence à penser à aller pêcher à l'extérieur du Mali. À partir de 1966, il multiplie les sorties internationales dans presque toute la sous-région, avant de se fixer définitivement au bord du lac avec sa famille. "Je me suis rendu en 1966, pour ma première sortie internationale, au Ghana pour trois ans; au Burkina en 1972 pour trois ans éga- 
lement ; en Sierra Leone en 1983 pour un an. Enfin, en 1984, je me suis retrouvé au Libéria durant un an, tout juste avant de venir m'installer définitivement ici à Sélingué, à Carrière en 1985."(Enquête Carrière)

Un autre de ces pêcheurs, âgé de 56 ans, déclare être originaire du village de Konakoro dans le cercle de Djenné. Polygame (quatre femmes), il est venu pour la première fois à Sélingué en 1984 en tant que migrant saisonnier. De 1984 à 1986, chaque année, il est venu passer cinq mois à Sélingué. En 1986, il s'est installé d'abord au campement de Carrière avant d'aller à celui de Barkabougou en 1987. Depuis ses 16 ans, il rejoignait chaque saison son oncle paternel à Ségou au bord du fleuve Niger, où celui-ci s'était établi. "Je venais faire la pêche auprès de mon oncle à Ségou chaque année, pendant six mois au maximum, avant de repartir chez mon père dans mon village." Quand il a eu 29 ans, son père l'a envoyé auprès de son cousin en Côte d'Ivoire, après lui avoir donné une femme. "J'y suis resté pendant une année et demie avant de venir prendre ma deuxième épouse au village." Après quelques mois auprès des siens, il décide de partir avec un ami jusqu'en Mauritanie, où il reste pendant neuf mois. En 1983, il effectue une dernière sortie à l'extérieur, au Ghana notamment, avant de retourner dans son village. Ce retour au village fut de courte durée, car il coïncida avec l'ouverture du barrage de Sélingué. De nos jours, sous le poids de l'âge, il ne peut plus voyager loin et a passé le flambeau à son frère, qui pratique la pêche au Burkina Faso, et à son fils aîné, qui, selon lui, serait en Côte d'Ivoire. "Je regrette de n'avoir été seulement qu'en Côte d'Ivoire, Mauritanie et Ghana, mais mon frère et mon fils, tant qu'ils resteront jeunes, iront partout où ils voudront pour faire la pêche.”(Enquêtes, Barkabougoudaga, octobre 2006)

\section{Migrer, une stratégie familiale}

La migration, quelle que soit sa nature, est souvent considérée comme une réponse individuelle plutôt passive, à la fois à la pauvreté rurale (facteur de répulsion) et au besoin des marchés de travail (facteur d'attraction). Pourtant, cette approche stratégique n'est pas purement individualiste, car elle se trouve systématiquement mise en relation avec la famille. En effet, dans les migrations de pêche à Sélingué, nous avons constaté que, même en se centrant sur l'individu (les parcours migratoires individuels), il est impossible d'échapper à la réalité collective (donc familiale) qui agit, le plus souvent, comme une ressource essentielle dans le processus migratoire. En facilitant, voire en organisant les départs, ou en constituant un réseau d'accueil ou d'entraide. Ainsi, au sein d'un même ménage, tous les frères peuvent migrer, mais ne le font pas en même temps. Une fois sur les lieux de pêche, un pêcheur retrouvera un frère ou un membre aîné de 
sa famille, sous la tutelle duquel il pourra se placer pendant la durée de son séjour. Les entretiens avec les pêcheurs ont révélé que chaque migrant a au moins un parent en migration, à l'intérieur comme à l'extérieur. Ce terme de "parent"est pris, ici, dans son sens le plus large, le plus élastique, et peut désigner de ce fait un fils, un frère, un oncle, un cousin, un père, etc. D'où le rôle déterminant de la famille dans l'organisation des départs et des accueils.

\section{L'influence du faso : le contrôle des migrants par les parents}

Cette notion de famille, du groupe familial d'appartenance, a une grande influence sur l'inconscient collectif des pêcheurs, au point qu'ils se trouvent, le plus souvent, sous son contrôle. Il est très rare de soumettre un pêcheur à un entretien sans qu'il n'évoque un parent, un frère, un cousin, un oncle... dont le rôle a été déterminant dans son départ tout comme dans son accueil. Ainsi, le pêcheur migrant se trouve dans une situation où le faso, c'est-à-dire le groupe d'appartenance, le village natal, exerce sur lui et sur ses biens, même à distance, une certaine pression. Depuis plusieurs décennies, la monétarisation des rapports de production n'est pas étrangère à cette nouvelle situation. Mais le désir réel d'émancipation des jeunes intervient amplement et les fait opter ainsi, de plus en plus, pour des migrations lointaines et prolongées. Bréhima Kassibo considère que la migration des jeunes pêcheurs hors du delta les éloigne des contraintes du pouvoir gérontocratique. Selon lui, "une fois soustraits aux obligations coutumières, les cadets - surtout ceux qui ne pourront accéder rapidement à une situation statutaire dominante dans leur famille - ont tendance à ne plus retourner au village. Ils diminuent les rythmes des visites et les envois d'argent au village. Cependant, les aînés parviennent toujours à les localiser, même à l'étranger, et il n'est pas rare de les voir débarquer dans les campements de pêche pour réclamer le montant de l'impôt du faso aux récalcitrants, et les rappeler à l'ordre le cas échéant $t^{(6)}$.

\section{Le mariage, des obligations sociales au commerce}

A. M. Jeay note qu'à l'époque des premières migrations les hommes partaient seuls : "Les pêcheurs n'amènent jamais leur femme à l'étranger"." Mais, depuis deux décennies, après un mariage célébré au village natal, les pêcheurs retournent en migration avec leur épouse, ou les font envoyer par leur chef de ménage, même s'ils ont commencé à 
migrer en étant célibataires. Pour ce qui est des pêcheurs migrants de Sélingué, nous avons constaté que le mariage constitue réellement un puissant moyen de contrôle social sur eux. L'endogamie étant répandue chez les pêcheurs bozo et somono, les jeunes migrants célibataires se marient très rarement à l'étranger. Ils vont tous prendre femme chez eux, parmi les leurs, seuls habilités à bénir les unions. C'est toujours le groupe familial qui procure une épouse. Et cette alliance matrimoniale, contrôlée par les aînés des lignages des deux conjoints, se révèle un moyen de pression sur le jeune migrant à travers les devoirs sociaux qu'elle crée, et dont l'observance est obligatoire. À Sélingué, le rôle des femmes est déterminant dans la commercialisation du poisson de leurs époux, même si elles ne vont jamais à la pêche. Commercialisée à partir des deux principaux débarcadères (Carrière et Faraba), la plus grande partie de la production halieutique du lac est drainée vers Bamako, la capitale, par l'intermédiaire des mareyeuses. Les femmes grossistes et revendeuses du poisson frais dominent toute la filière. Elles emploient une main-d'ceuvre locale (contre rémunération) souvent constituée de femmes de pêcheurs qui s'occupent de la réception, du lavage et du conditionnement du poisson. En alternance avec des couches de glace, le poisson frais est emballé dans de gros paniers en bambou (en moyenne 0,90 m de diamètre et $0,40 \mathrm{~m}$ de hauteur), tapissés à l'intérieur de jute. Ces paniers pouvant contenir chacun 130 à $240 \mathrm{~kg}$ de poisson, selon leur dimension, sont chargés dans des camionnettes affrétées par les mareyeuses et expédiés à Bamako pour être vendus sur les marchés de Dibida et de Médina coura.

Sur place, pas plus du tiers de la production du lac est commercialisé par les femmes de pêcheurs sur les foires locales hebdomadaires, à Kangaré ou à la cité administrative. Si tout le produit à l'état frais n'est pas commercialisé, le processus continue par sa transformation : fumage, séchage, ou (rarement) brûlage, qui sont les principales techniques de conservation.

\section{Le retour au village ou le poids de la coutume}

Toutes les occasions sont bonnes pour un pêcheur migrant de retourner chez lui ou d'entrer en contact avec les siens : visites annuelles, envois de nouvelles, de cadeaux, d'argent ou de matériels de pêche, etc. Pour une bonne partie des pêcheurs, même installés de façon permanente, à l'occasion des décès de proches parents du couple au village, le mari et la femme ont l'obligation de venir présenter leurs condoléances. Un dernier lien des migrants avec leur village d'origine est le retour définitif des vieux pêcheurs pour succéder à leurs frères aînés, une fois décédés, dans la chefferie et la gestion du ménage ou du village. 
Seuls le temps et le statut du migrant peuvent jouer en faveur d'un relâchement total ou partiel des rapports familiaux, mais la force d'attraction du faso est très grande sur des individus soucieux d'éviter une perte d'identité ou l'exclusion du groupe d'appartenance. Les aînés sont conscients de la nécessité que représente la manne migratoire. Ils exercent une pression idéologique permanente sur les membres de leur groupe d'appartenance partis en migration, en vue de les maintenir sous leur autorité. L'analyse de ces formes de contrôle social montre que la vie sociale repose sur un ensemble structuré de formes d'emprises que les hommes exercent les uns sur les autres. On peut d'ores et déjà estimer à partir de là que les migrations de pêche ont toujours existé, quoique de façon bien moins importante qu'aujourd'hui.

\section{Conclusion}

Les migrations de pêche ont ceci d'intéressant qu'elles font partie de ces comportements spontanés non imputables aux interventions extérieures. C'est le développement de la production et la monétarisation du secteur qui peuvent expliquer leur accroissement. Certaines interprétations des phénomènes de migration ont mis l'accent, unilatéralement par le passé, sur des "causes culturelles ${ }^{(8)}$. Il ne faudrait pas commettre l'erreur inverse et n'y voir que l'effet des seuls mécanismes économiques (cf. Thomas M. Painter, 1987). Elles résultent de phénomènes sociaux complexes qui sont le produit de facteurs de même nature. Donc une analyse de la migration ne peut en aucun cas être monocausale.

Les migrations combinent la recherche du numéraire, l'émancipation des cadets, la quête du prestige (dans le cas des migrations internationales), etc. On y voit à l'oeuvre, imbriqués et en partie indiscernables, aussi bien des moyens de pourvoir aux besoins familiaux ou individuels que des modes de reconnaissance sociale, ou l'accès à de nouveaux réseaux de sociabilité.

Mais dans tous les cas de figure, que la migration soit interne ou externe, les pêcheurs restent et demeurent attachés à leur origine, à leur famille et à leur village natal. C'est pourquoi tout déplacement de courte, moyenne ou longue durée est toujours conclu par un retour dans la famille. Dans les cas d'une sédentarisation dans les lieux de migration, comme c'est de plus en plus le cas à Sélingué, les liens avec le faso se traduisent par des visites, des envois d'argent aux parents restés au village, comme pour leur signifier que, malgré la distance, ils ne sont point oubliés. Ainsi, les pêcheurs partis en migration demeurent sous l'influence du faso. Ils continuent de jouer un rôle dans la reproduction du système social. 
Notes

1. Au Mali, le Delta central représente une vaste cuvette d'inondation d'une superficie de $30000 \mathrm{~km}^{2}$ environ dans les années de fortes crues. Il possède un réseau hydrographique dense et varié, constitué des fleuves Niger, Sénégal et leurs nombreux affluents. En plein centre du pays, dans la région de Mopti, le fleuve Niger se jette dans une dépression appelée le Delta central (intérieur). Il s'étend de Markala (Ségou, 4 e région administrative du pays), en passant par Mopti ( $5^{\mathrm{e}}$ région), jusqu'à Tombouctou ( $6^{\mathrm{e}}$ région). En plus de la pêche, cet écosystème particulier se prête à d'autres activités tels que l'élevage et la riziculture, et la pratique de ces trois activités depuis des siècles lui a conféré une place de choix dans les activités économiques du pays.

2. Au Mali, les Malinké sont les gens du Mandé dont l'orthographe utilisée ici correspond aux normes dernièrement définies. On peut, cependant, indifféremment, trouver dans les différentes publications les orthographes suivantes: Mandé, Manden, Manding. "Ce terme fait allusion au groupe linguistique mandé qui recouvre une grande partie des savanes et de la zone forestière de l'Afrique occidentale. Il désigne également les populations issues de l'Empire du Mali (qui atteint son apogée au XIV e siècle) et celles qui ont subi son influence sur le plan sociopolitique, économique et religieux." (Z. Clemens). La zone de Kangaba est considérée comme le principal foyer du Mandé au Mali. 3. Étude socio-économique conjointement réalisée par la Direction nationale des ressources forestières et hydrauliques (DNRFH), le Programme d'appui à la mise en ceuvre du schéma directeur du secteur du développement rural (PAMOS) et la Division développement rural (DDR) de l'ex-OERHN de Sélingué, sur financement FAO, Bamako, 1995.

4. Le barrage est une technique de pêche qui s'effectue sur les bras ou les couloirs du lac et qui consiste, littéralement, à "barrer" le passage des poissons à l'aide d'un long filet tendu entre deux piquets, de sorte que les poissons au cours de leur déplacement entre les bouts du couloir viennent se mailler dans le filet, qui en fait "coupe" le couloir en deux côtés. 5. La population des pêcheurs, selon un recensement de la Division Pêche Environnement (DPE) de l'ODRS (1998), s'élevait à 6991 personnes dont 3123 actifs, repartis entre 741 ménages et vivant dans 71 campements permanents. Une année plus tard, la même population s'élève à 8869 personnes dont 6496 actifs opérant sur les eaux du lac. En 2001, selon le même recensement, cette population connut une légère baisse puisqu'elle revient à 6734 personnes dont 4116 actifs. Cette fluctuation s'explique par le fait que les pêcheurs diversifient de plus en plus leurs itinéraires de migration et le lac de Sélingué, après la faste décennie quatre-vingt-dix, est de moins en moins le premier choix des pêcheurs migrants comme site de destination. La pression démographique et son corollaire, l'intensification accrue de l'effort de pêche, entraînent une diminution notable de la ressource et une certaine rigidité de la réglementation d'accès à cette même ressource. Les débuts de l'application de la convention locale de pêche rétrécissent les marges de manceuvre des pêcheurs avec l'apparition des règles de pêche sur le lac. On note ainsi un abandon progressif des techniques de pêche dites nocives, dont le barrage.

6. Bréhima Kassibo, "Pêche continentale et migration : contrôle politique et contrôle social des migrations de pêche dans le delta central du Niger (Mali)", in J. P. Chauveau, E et E. Y. Larsen (édit.), Les pêches piroguières en Afrique de l'ouest : pouvoir, mobilité, marché, Paris, Karthala, 2000, p.244.

7. A. M. Jeay, "Prédateurs de la nuit, études ethnosociologiques d'une population de pêcheurs : les Somono (Mali)", Thèse d'état en ethnologie, Montpellier 3, 2vols, 1984.

8. cf. Jean Rouch, Migrations au Ghana, Gold Coast, enquêtes 1953-1956, Paris, Société des Africanistes, 1956.

\section{Bibliographie}

- J.P. Chauveau, Histoire de la pêche maritime et politique de développement de la pêche en général. Représentations et politiques du dispositif de l'intervention moderniste. Anthropologie maritime 2, 1985, p.300-318.

- J.P. Chauveau, Histoire de la pêche industrielle au Sénégal et politiques d'industrialisation, Cahiers des Sciences Humaines 25, 1989, p.237-287.

- Tata Youssouf-Wâ Kamissoko Cissé, La grande geste du Mali. Des origines à la fondation de l'empire, Karthala-Arsan, Paris, 1988.

- Claude Fay, "Des poissons et des hommes : pêcheurs, chercheurs et administrateurs face à la pêche au Maacina (Mali)" in J.P. Chauveau, E. J. Larsen et C. Chaboud (édit), La pêche piroguière en Afrique de l'Ouest. Pouvoirs, mobilités, marchés. CMI-IRD-KARTHALA, 2000.

- J. Gallais, Le delta intérieur du Niger. Etude de géographie régionale, Dakar, IFAN, 2 vols, 1967.

- R. Lae, Les pêches artisanales lagunaires ouest africaines. Echantillonnage et dynamique de la ressource et de l'exploitation, ORSTOM-Bondy, 1992.

- Ministère du Développement Rural et de l'Environnement, Direction Nationale de l'Aménagement et de l'Equipement Rural, Revue du secteur de la pêche et de la pisciculture, Avril 1997

- OEHRN, Impact socio-économique du barrage sur son environnement immédiat. Juillet 1980, Sélingué, 27 pages.

- OEHRN, Plan d'exécution du transfert des populations touchées par le barrage de Sélingué. Rapport final, 1980, Tome VIII.

- OERHN, Direction des études et de la programmation : Enquête socio-économique en milieu pêcheur, DPE, juillet 1985.

- Jean Rouch, Les hommes et les dieux du fleuve. Essai ethnographique sur les populations songhay du moyen Niger 1941-1945, ART COM, Paris, 1997.

- Jean Yves Weigel (dir.), La pêche en Afrique : enjeux et défi. Afrique Contemporaine, nº 187, juillet septembre, 1998. 